\title{
Quantitative evaluation in combination with nonquantitative evaluation in early patellar cartilage osteoarthritis at $3.0 \mathrm{~T}$
}

This article was published in the following Dove Press journal:

Clinical Interventions in Aging

16 July 2014

Number of times this article has been viewed

\author{
Houdong Zuo' \\ Weiwu Yao' \\ Nan Qu' \\ Shixun Yang' \\ Jianhua Wang ${ }^{2}$ \\ Xiaojiang $\mathrm{Cui}^{3}$ \\ 'Department of Radiology, Shanghai \\ Jiao Tong University Affiliated Sixth \\ People's Hospital, Shanghai, People's \\ Republic of China; ${ }^{2}$ Department \\ of Orthopedics, Xin Hua Hospital \\ Affiliated to Shanghai Jiao Tong \\ University School of Medicine, \\ Shanghai, People's Republic of China; \\ ${ }^{3}$ Department of Surgery, Department \\ of Obstetrics and Gynecology, Samuel \\ Oschin Comprehensive Cancer \\ Institute, Cedars-Sinai Medical Center, \\ Los Angeles, CA, USA
}

Purpose: To evaluate quantitative $\mathrm{T} 1$ and $\mathrm{T} 2$ relaxation times and magnetization transfer ratios (MTRs) in the early diagnosis of patellar cartilage osteoarthritis (OA) and to quantify and possibly refine the current Kellgren-Lawrence score criteria.

Materials and methods: A total of 92 cases of knee joints with 40 normal volunteers and 30 patients with OA were prospectively evaluated. The knee joints with OA were divided into mild and moderate groups according to the Kellgren-Lawrence score criteria. The discriminative analysis method was used to analyze the accuracy of the original grouped cases correctly classified by age, sex, T1 relaxation times, T2 relaxation times, and MTR values. Linear regression analysis was used between T1 relaxation time, T2 relaxation time, and MTR values.

Results: The mean $\mathrm{T} 1$ relaxation times decreased with the severity of OA, and a significant difference was only found between the normal and moderate OA groups $(P<0.05)$. The mean $\mathrm{T} 2$ relaxation times increased, and significant differences were found between the normal and mild OA groups and the normal and moderate OA groups $(P<0.001)$. The MTR values were $35.8 \% \pm 4.2 \%, 36.1 \% \pm 3.2 \%$, and $35.4 \% \pm 3.8 \%$, respectively. There were no significant differences between the normal and OA groups. In addition, T1 relaxation times were positively correlated with MTR values $(P<0.01)$. A discriminative analysis using a synthesis of all the influential factors indicated a high accuracy rate $(93.9 \%)$ for the correct classification of the original grouped cases.

Conclusion: Quantitative T1 and T2 relaxation times were useful in the diagnosis of early OA; T2 relaxation times were more relatively sensitive. The functional usefulness of MTR values may be limited. T1 relaxation times positively correlated with MTR values. Multiple quantitative parameters, combined with some relative nonquantitative clinical parameters and Kellgren-Lawrence scores, may be useful in the early stage of OA and provide better information for clinical treatment and follow-up.

Keywords: osteoarthritis, patellar cartilage, magnetic resonance imaging, T1 relaxation time, T2 relaxation time, MTR, age, sex

\section{Introduction}

Osteoarthritis (OA) is one of the most common musculoskeletal diseases. It produces a series of clinical symptoms including pain, dysfunction, joint deformity, and even joint stiffness and rigidity, and it affects millions of individuals. ${ }^{1,2}$ In the development process of OA, articular cartilage changes play a key role, such as the loss of hyaline articular cartilage and the degradation of proteoglycans (PGs). ${ }^{1,3}$. The cartilage consists of water, low-density chondrocytes, and a large amount of extracellular matrix. ${ }^{5}$ The matrix contains water and a cross-linked matrix with PGs and type 2 collagen fibers, and PGs consist mainly of glycosaminoglycans (GAGs). Articular cartilage is divided
Correspondence: Weiwu Yao Department of Radiology, Shanghai Jiao Tong University Affiliated Sixth People's Hospital, 600 Yishan Road, Shanghai 200233, People's Republic of China Tel +8602164369181

Fax +860216470 I36I

Email yaoweiwu@yahoo.com 
into four zones, superficial, intermediate, deep, and calcified, from the surface to the inner bone. Some researchers have reported that the depletion of PGs and the breakdown of collagen fibers with early OA are possibly involved with the composition and structure of the superficial cartilage. ${ }^{6-9}$ Biochemical alterations to the articular cartilage are more likely to occur before morphologic degradation. Therefore, early diagnosis of OA is important for clinical treatment. As a consequence, the quantitative evaluation of the biochemical compositional changes of cartilage is valuable for the early diagnosis of OA.

Magnetic resonance imaging (MRI) is a noninvasive and useful protocol that is sensitive and can detect the composition changes of cartilage degeneration. ${ }^{8-10}$ Several MRI techniques are available, including delayed gadolinium-enhanced MRI of the cartilage (dGEMRIC), T2 mapping, magnetization transfer (MT) imaging, T1 $\rho$ imaging, and sodium MRI. ${ }^{1-14}$ dGEMRIC is widely applied to detect the changes of GAGs with abundant negative-charge side chains that provide a negative fixed-charged density to the cartilage in the early stage of OA. Gd-DTPA ${ }^{2-}$ is a type of agent contrast with negative charge distributing in an opposite manner, with the GAGs in the cartilage. In normal cartilage, Gd-DTPA ${ }^{2-}$ is repelled by the GAGs; in contrast, under the conditions of GAGs loss, more Gd-DTPA ${ }^{2-}$ will be distributed within the cartilage matrix. ${ }^{8,11,15,16}$ T2 mapping is also widely used and has been proven to be more sensitive to collagen changes in the cartilage. ${ }^{8,13,17}$ The MT imaging technique is now accepted and used in articular cartilage. MT is tissue-specific and also can provide quantitative data. ${ }^{18-21}$ Nevertheless, MT imaging is still not widely used in articular cartilage studies. T1 $\rho$ can reflect the changes of GAGs and collagen, but several factors may affect the T1 $\rho$, leading to variations of $\mathrm{T} 1 \rho .{ }^{12,13,22} \mathrm{~T} 1 \rho$ has the drawback of relatively high specific absorption rate problems and potential tissue-heating effects. Sodium MRI provides an additional technique for quantifying the biochemical compositions of cartilage on the basis of similar principles to dGEMRIC; however, it is difficult to produce an magnetic resonance (MR) signal with sodium because ${ }^{23} \mathrm{Na}$ ions exist at much lower concentrations than ${ }^{1} \mathrm{H}$ ions within the body, which limits their application in cartilage. ${ }^{23}$

The use of a panel of biomarkers for quantification is certainly the next step in quantitative MRI. Thus, in this study, the quantitative parameters of $\mathrm{T} 1$ and $\mathrm{T} 2$ relaxation times were evaluated. Moreover, the quantitative parameters and nonquantitative factors were also investigated. Using this method, the current nonquantitative Kellgren-Lawrence (K-L) score criteria were expected to be quantified and refined.
To our knowledge, this was the first study examining these particular evaluations.

\section{Materials and methods Subjects}

A total of 36 men and 34 women with a total of 92 knee joint cases were enrolled in this study. Written informed consent was obtained from all the participants before the study, according to the requirements of the review board of Shanghai Jiao Tong University, Affiliated Sixth People's Hospital. The participants consisted of 40 volunteers with a mean age of 27.6 \pm 11.5 years (range, 14-61 years), 16 patients with mild $\mathrm{OA}$ with a mean age of $47.8 \pm 11.2$ years (range, 26-71 years), and 14 patients with moderate OA with a mean age of $61.0 \pm 7.3$ years (range, $50-80$ years). Total knee joints were evaluated with a 3.0 T MR imaging spectrometer between December 2012 and July 2013 and were categorized by radiographs and clinical history.

Inclusion criteria for the normal volunteer group were no history of severe trauma and surgery, Western Ontario and McMaster Universities OA index ${ }^{24}$ score lower than 10 points, and a body mass index ranging from 18 to $25 \mathrm{~kg} / \mathrm{m}^{2}$. Exclusion criteria were knee joint pain or discomfort, contraindications, and severe cardiovascular or mental diseases with MRI examinations.

As in earlier surveys OA was divided into five grades, as follows: none (0), doubtful (1), minimal (2), moderate (3), and severe (4). ${ }^{25}$ In a previous study by our team, Yao et $\mathrm{al}^{26}$ investigated the application of $\mathrm{T} 1$ relaxation time, T2 relaxation time, and magnetization transfer ratios (MTR) in the early diagnosis of patellar cartilage OA. A small number of cases was involved, so further research with a larger number of participants was necessary for reproducibility. In this study, the inclusion criteria for mild OA patients were K-L grades 1 and 2 , and for moderate OA, K-L grade 3 . The severe OA group (K-L grade 4) was not enrolled into our study because of severe depletion of PG and collagen, dehydration, extensive fibrillation, and denudation of the subchondral bone, which would greatly influence the results. ${ }^{5,6,27}$

\section{MR imaging protocols}

All patients were examined in a Philips Achieva 3.0 T superconducting MR scanner (Koninklijke Philips NV, Amsterdam, the Netherlands) with an eight-channel high-resolution knee joint coil in the study. The T2W sampling array used an eight-echo spin-echo (SE) sequence. The relative parameters were as follows: repetition time (TR), 2,100 ms; echo time (TE), 15 130 ms; slice thickness, $3 \mathrm{~mm}$; field of view 
(FOV), 16 mm; matrix size, $332 \times 273$; startup echoes, 1; and scanning time, 5 minutes. Sagittal and axial short-time inversion recovery $(\mathrm{TR} / \mathrm{TE}=3,000 \sim 4,000 \mathrm{~ms} / 41.3 \sim 48 \mathrm{~ms}$; inversion time (TI), $150 \mathrm{~ms}$ ), T1-weighted fast SE sequences ( TR/TE $=500 / 20 \mathrm{~ms}$ ), and T2-weighted fast SE sequences ( $\mathrm{TR} / \mathrm{TE}=3,890 / 100 \mathrm{~ms}$ ) were used. The morphology and patellar cartilage signal characteristics were evaluated.

All images were sampled simultaneously and processed at a workstation (ADW 4.0; GE Healthcare Bio-Sciences Corp., Piscataway, NJ, USA) with equipped software (Philips). The cartilage contour in the central plane axial view was outlined manually three times, and the mean $\mathrm{T} 2$ relaxation time was also measured three times.

Magnetization transfer images and MTR values were obtained using a three-dimensional dynamic gradient echo sequence. The main parameters included a deviation frequency of $1,100 \mathrm{~Hz}$, a radiofrequency angle of $520^{\circ}$, a TR of $66 \mathrm{~ms}$, a TE of $3.8 \mathrm{~ms}$, a slice thickness of $1 \mathrm{~mm}$, a FOV of $16 \mathrm{~cm}$, a matrix size of $160 \times 132$, and a scanning time of 1 minute 37 seconds. The cartilage contour in the central plane axial view was outlined, and the MTR values were measured three times.

For dGEMRIC assessment, the subjects were injected intravenously with $0.2 \mathrm{mmol} / \mathrm{kg}$ Gd-DTPA ${ }^{2-}$ contrast agent (Magnevist $^{\circledR}$; Bayer Schering Pharma AG, Berlin-Wedding, Germany) and asked to walk or perform some exercises for 10 minutes to aid the in diffusion of the contrast agent into the cartilage. After 1.5 hours of Gd-DTPA ${ }^{2-}$ administration, the three-dimensional look locker pulse sequence technique was applied to each knee joint. Each reverse recovery curve collected $30 \mathrm{TI}$, and central phase encoding was applied for each slice. The first TI was $60 \mathrm{~ms}$, and the other TI times were $60 \mathrm{~ms}+\mathrm{TR} \cdot \mathrm{n} \cdot$ \#slice, $\mathrm{n}=1-29 .{ }^{26}$ Other parameters include the following: TR, $20 \mathrm{~ms}$; TE, $3.9 \mathrm{~ms}$; FOV, $16 \mathrm{~cm}$; matrix size, $152 \times 115 ; \alpha, 10^{\circ}$; bandwidth, $189.1 \mathrm{KHz}$; and scanning time, 6 minutes and 30 seconds plus. These data were sent to the workstation (ADW 4.0), and colorful T1 images were produced, using Philips T1 imaging software. The central plane contour of the patellar cartilage was outlined, and the mean $\mathrm{T} 1$ relaxation time was measured.

\section{Statistical analysis}

Continuous variables are presented as mean \pm standard deviation. Quantitative evaluation was processed with one-way analysis of variance.

Categorical variables were analyzed with a chi-squared test. Multivariate analysis with a linear regression model (correlation among $\mathrm{T} 1$ relaxation time, T2 relaxation time, and MTR values) was performed. The canonical discriminant function, which was expressed as $U_{k m}(X)=u_{1} X_{1 \mathrm{~km}}$ $+u_{2} X_{2 k m}+\cdots u_{i} X_{i k m}+a=u^{\prime} X$, was used, where $\mathrm{u}=\left(u_{1}, u_{2}, \cdots, u_{i}\right)^{\prime}$ are the discriminative coefficients that produce the desired characteristics of the function, $U_{k m}$ is the value (score) of the canonical discriminant function for case $m$ in group $\mathrm{k}$, $X_{i k m}$ is the value for the discriminating variable $X_{i}$ for case $m$ in group $k$, and $a$ is a constant. The formula is based on the principle that a batch of samples is classified into the appropriate group according to the calculated scores. The discriminant score is a weighted linear combination (sum) of the discriminating variables. All tests for statistical significance were two-tailed, with an $\alpha$ level of 0.05. All analyses were conducted with SPSS 19.0 software for Windows (IBM Corporation, Armonk, NY, USA).

\section{Results}

\section{Imaging analysis}

On dGEMRIC, the normal cartilage located between the red and orange color steps was integrated, and the signal was homogeneous (Figure 1A). The morphology with mild OA was similar to the normal cartilage; where the signal was slightly heterogeneous, a bronze color appeared (Figure 1B). The moderate OA cartilage was localized and thin, with a crimson or bronze abnormal signal in the cartilage. The joint space was mildly narrow (Figure 1C).

On the T2 mapping and Magnetization Transfer Contrast (MTC) images, the normal cartilage contour was integrated and continuous, without marked thinness. The signal was homogeneous, and the joint space was normal (Figure 2A and Figure 3A). With mild OA cartilage, the cartilage was still integrated and continuous, without marked thinness. However, some patchy or mottled low-signal areas appeared (Figure 2B, C and Figure 3B). The joint space was normal or doubtfully narrow, whereas localized thin and irregular morphology could be found in the cartilage with moderate OA. The signal was heterogeneous and patchy, and striped lower-signal areas appeared in the center or the lateral sides of the cartilage (Figure 2D-F and Figure 3C). The joint space was mildly or moderately narrow.

\section{Evaluation of $\mathrm{TI}$ relaxation times}

The mean T1 relaxation times of the normal, mild OA, and moderate OA groups were 481.7 $\pm 66.9,478.3 \pm 64.5$, and $407.0 \pm 42.3 \mathrm{~ms}$, respectively. With the worsening degeneration of articular cartilage, the $\mathrm{T} 1$ relaxation times became lower. The values dropped drastically between the mild OA group and the moderate OA group. Significant 

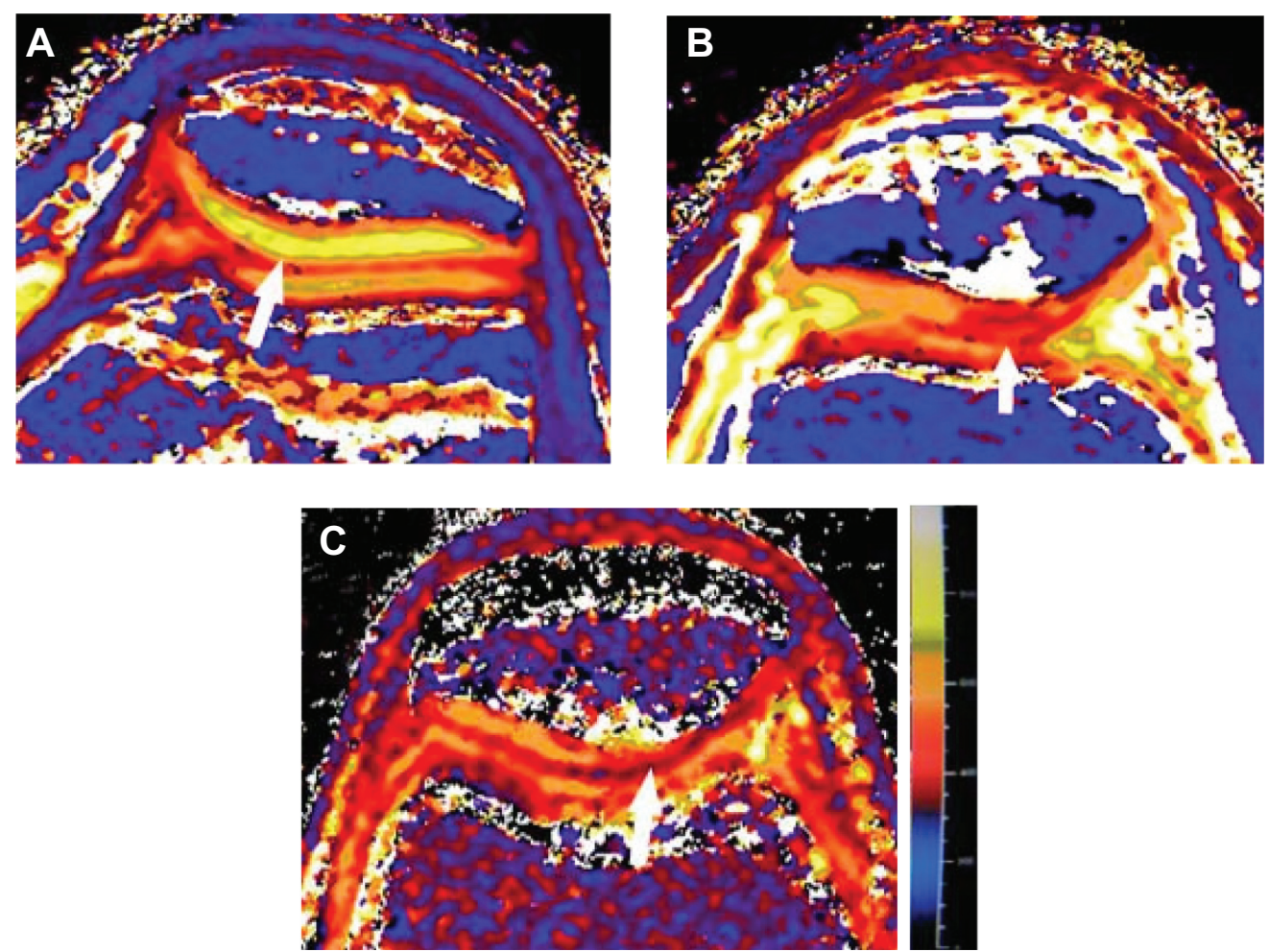

Figure I TI imaging of patellar cartilage.

Notes: (A) Normal cartilage is located between the red and orange color steps. The contour is integrated and regular, and the signal is homogeneous (white arrow). (B) Imaging of the cartilage with mild osteoarthritis. The contour is still integrated but slightly irregular, and the bronze color appears (white arrow). (C) Imaging of the cartilage with moderate osteoarthritis. The local cartilage becomes clear. The contour is irregular, and some bronze area appears. The joint space is becoming narrow (white arrow).

differences were found only between the normal and moderate groups $(P<0.05)$. No significant differences were found in the other two combinations $(P>0.05$; Table 1$)$. These results suggest that $\mathrm{T} 1$ relaxation times were sensitive to the changes of GAGs in the moderate stage of OA.

\section{Evaluation of T2 relaxation times}

The mean $\mathrm{T} 2$ relaxation times of the normal, mild OA, and moderate OA groups were $47.3 \pm 4.5,51.1 \pm 6.9$, and $53.9 \pm 9.2 \mathrm{~ms}$, respectively, measured on T2 mapping. $\mathrm{T} 2$ relaxation time increased with the degree of $\mathrm{OA}$, and the mild OA and moderate OA groups displayed significant differences with the normal group $(P<0.001)$, but no significant difference was found between the mild and moderate OA groups ( $P>0.05$; Table 1$)$. This demonstrates that the $\mathrm{T} 2$ relaxation times could sensitively reflect the early changes of collagen and are thus useful in clinical treatment.

\section{Evaluation of MTR values}

The mean MTR values of the normal, mild OA, and moderate groups were $35.8 \% \pm 4.2 \%, 36.1 \% \pm 3.2 \%$, and $35.4 \% \pm 3.8 \%$, respectively. No significant differences were found among these three groups regarding the mean MTR of patellar cartilage at the central plane $(P>0.05)$, with one-factor analysis of variance. In addition, no great variation appeared with an increased degree of OA. The mild OA group had a slightly higher value than that of the normal group, whereas the moderate OA group value was slightly lower than the normal and mild OA group values (Table 1). These results may indicate that MTR has some limits in the evaluation of early OA.

\section{Correlation among the quantitative parameters}

We also compared the correlation among these three quantitative parameters with a linear regression model and found that $\mathrm{T} 1$ relaxation times had a significant positive linear correlation with MTR values $(P<0.05)$, which indicates that the lower T1 relaxation time was related to the decreased MTR values, although there was no tendency toward a decrease in MTR values (Tables 2 and 3).

\section{Accuracy rate of the original grouped cases correctly classified, with discriminative analysis}

In this study, we used the quantitative parameters of T1 relaxation times, T2 relaxation times, and MTR values plus the 

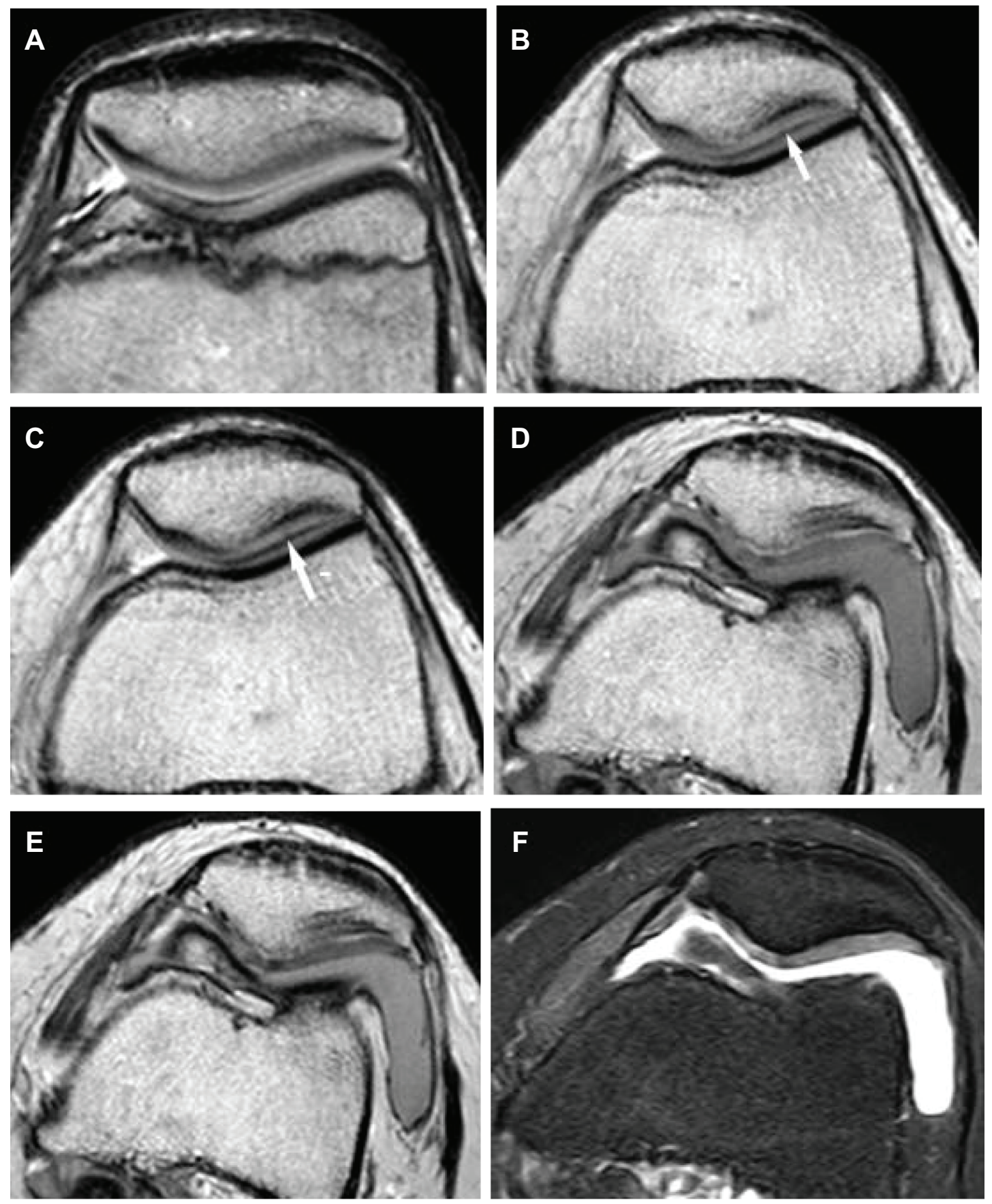

Figure $2 \mathrm{~T} 2$ imaging of patellar cartilage.

Notes: (A) An 18-year-old man. The patellar cartilage is integrated and clear (T2 mapping, TE $=15 \mathrm{~ms}$ ). (B and C) A 45-year-old woman. The patellar cartilage is still integrated and clear, but patchy low signals appear in the cartilage (T2 mapping: (B), TE $=15 \mathrm{~ms} ;(\mathbf{C}), \mathrm{TE}=30 \mathrm{~ms}$ ) (white arrows). (D-F) A 53-year-old woman. The cartilage is irregular, and the signal is heterogeneous and patchy. Striped lower signal areas can be seen (T2 mapping; (D), TE =15 ms; (E), TE =30 ms). These abnormal signals can be demonstrated using T2 SE sequence imaging (F).

Abbreviations: SE, spin-echo; TE, echo time.

factors of age and sex to analyze the accuracy of the correct classification (\%) of the original grouped cases based on nonquantitative $\mathrm{K}-\mathrm{L}$ grade criteria. The highest accuracy reached $93.9 \%$ when all the factors of $\mathrm{T} 1$ relaxation time, T2 relaxation time, MTR values, age, and sex were taken into consideration. The result indicated that the application of these quantitative parameters in OA could quantify the $\mathrm{K}-\mathrm{L}$ criteria. What is more important is that some quantitative indexes could be applied in clinic. We also could see that the factors of $\mathrm{T} 1$ relaxation time and sex were dominant. In clinical settings, we can use these parameters and factors in combination with K-L score criteria to evaluate early OA 

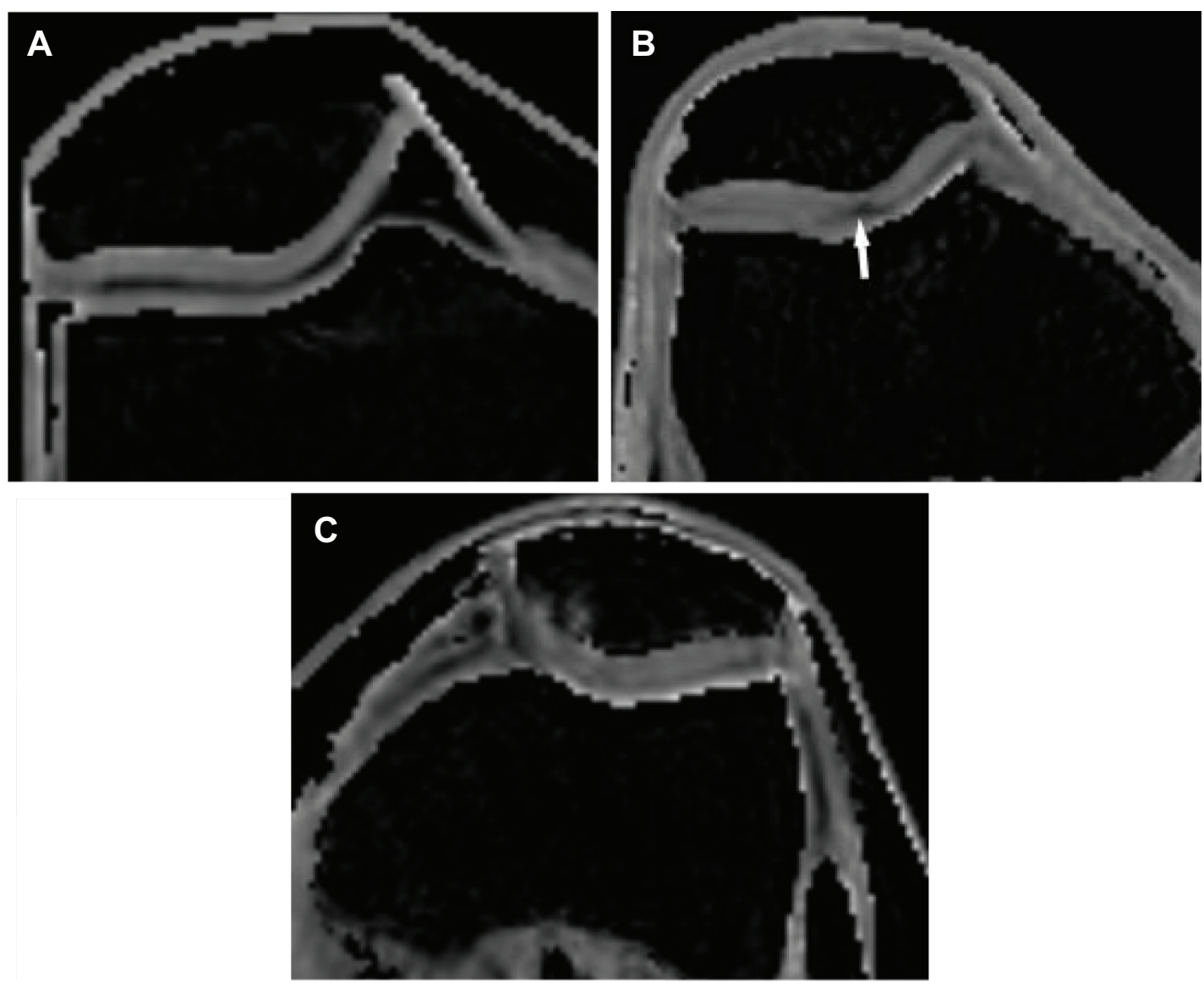

Figure 3 Magnetization Transfer Contrast (MTC) subtraction images.

Notes: (A) A 26-year-old woman. Imaging of normal patellar cartilage. The cartilage is integrated and continuous, and the signal is homogeneous. (B) A 60-year-old man. Imaging of patellar cartilage with mild osteoarthritis. The cartilage is still integrated and continuous, but patchy and striped low signals appear in the cartilage (white arrow). (C) A 56-year-old man. Imaging of patellar cartilage with moderate osteoarthritis. The cartilage has become thin, and the morphology is irregular and uneven. Some patchy or irregular low areas are visible.

(Table 4). In addition, the current nonquantitative K-L score criteria can be refined, and new and quantitative criteria could be proposed (Table 5). The highlight of these new criteria is to enable the quantitative evaluation of normal and OA cartilage in the clinic. In this way, more useful information is offered for clinical treatment and follow-up visits with OA patients.

\section{Discussion}

In this study, three types of MR techniques were applied in combination. T1 and T2 mapping are currently widely used and have proven to be important in the evaluation of cartilage, but the MT technique has been relatively less studied. Therefore, the trial with three different techniques in this study was relatively novel and encouraging. We found that

Table I Demographic and clinical data for our study groups

\begin{tabular}{|c|c|c|c|c|}
\hline Characteristic & Normal $(n=50)$ & Mild $(n=22)$ & Moderate $(n=20)$ & $P$-value \\
\hline Age & $27.6 \pm 11.5$ & $47.8 \pm 11.2^{\dagger}$ & $61.0 \pm 7.3 *, \dagger$ & $<0.00 I^{\ddagger}$ \\
\hline Sex & & & & $0.001^{\ddagger}$ \\
\hline Male n (\%) & $28(70.0)$ & $5(3 \mid .3)$ & $3(21.4)$ & \\
\hline Female $\mathrm{n}(\%)$ & $12(30.0)$ & II (68.7) & II (78.6) & \\
\hline $\mathrm{TI}$ relaxation time & $481.7 \pm 66.9$ & $478.3 \pm 64.5$ & $407.0 \pm 42.3^{\dagger}$ & $<0.05^{\ddagger}$ \\
\hline $\mathrm{T} 2$ relaxation time & $47.3 \pm 4.5$ & $51.1 \pm 6.9^{\dagger}$ & $53.9 \pm 9.2^{\dagger}$ & $<0.00 I^{\ddagger}$ \\
\hline Magnetization transfer ratio, \% & $35.8 \pm 4.2$ & $36.1 \pm 3.2$ & $35.4 \pm 3.8$ & $>0.05$ \\
\hline
\end{tabular}

Notes: Continuous data compared with one-way analysis of variance for categorical variables with chi-squared test. *Significantly different compared with the normal group, as determined using analysis of variance test. ISignificantly different compared with the mild osteoarthritis group, as determined using analysis of variance test. 狩tatistical significance, $P<0.05$. 
Table 2 Multivariate analyses for factors influencing magnetization transfer ratio with linear regression analysis

\begin{tabular}{lllllr}
\hline Model & $\begin{array}{l}\text { Unstandardized } \\
\text { coefficients beta }\end{array}$ & $\begin{array}{l}\text { Coefficients } \\
\text { standard error }\end{array}$ & $\begin{array}{l}\text { Standardized } \\
\text { coefficients beta }\end{array}$ & $\boldsymbol{t}$-value & $\boldsymbol{P}$-value \\
\hline Constant & 0.113 & 0.057 & & 1.981 & 0.057 \\
TI relaxation time & 0.000 & 0.000 & 0.576 & 3.736 & 0.001 \\
Age & 0.002 & 0.000 & 0.597 & 3.305 & 0.003 \\
Sex & -0.035 & 0.017 & -0.378 & -2.080 & 0.046 \\
\hline
\end{tabular}

Note: The dependent variable is the magnetization transfer ratio.

$\mathrm{T} 1$ and $\mathrm{T} 2$ relaxation times were useful in the diagnosis of early $\mathrm{OA}$, and $\mathrm{T} 2$ relaxation times were relatively more sensitive. The usefulness of MTR may be limited. We also used the quantitative parameters and some relevant patient information to quantify and refine the current K-L score criteria. In addition, we also found the $\mathrm{T} 1$ relaxation times and MTR values correlated positively well, which is a new discovery.

$\mathrm{T} 2$ relaxation times are related to the loss speed of phase coherence and reflect the specificity of the tissue by describing the attenuation of transverse magnetization and the MR signal of the tissue. ${ }^{28}$ At this time, T2 relaxation mapping is a commonly used tool and has become a noninvasive biomarker to detect early cartilage degeneration, mainly including collagen matrix disarrangement and water increase in cartilage. ${ }^{29,30}$ In the early stages of OA, the matrix begins to break down and the water increasingly permeates the cartilage, leading to the enhancement of $\mathrm{T} 2$ relaxation times. ${ }^{31} \mathrm{In}$ recent studies, some researchers have reported that $\mathrm{T} 2$ relaxation times might also be sensitive to PG content, ${ }^{32-34}$ but the sensitivity of T2 to collagen is well-recognized.

Mosher et $\mathrm{al}^{30,35}$ report that the changes in age-related $\mathrm{T} 2$ relaxation may be related to senescent denaturation of the collagen fiber matrix. Cartilage changes in early OA may occur primarily and mainly in the superficial layer, where the collagen matrix first degenerates. ${ }^{36} \mathrm{Kim}$ et al ${ }^{37}$ report T2 mapping was correlated with histological degeneration, so T2 mapping may be a good biomarker for OA in human articular cartilage and may be more appropriate for the initial diagnosis of articular cartilage degeneration in the knee joint. Established animal models and in vivo studies have demonstrated that $\mathrm{T} 2$ values increase with severe cartilage damage or degeneration..$^{13,17,38}$

Dunn et a ${ }^{39}$ report significant differences between normal groups and mild or severe OA groups; however, there was no significant difference between the mild and severe OA groups. Prasad et $\mathrm{a}^{40}$ found that $\mathrm{T} 2$ values become higher with the progression of knee OA, but no significant differences in $\mathrm{T} 2$ values were found when assessing the progression cohort subgroups with incident lesions and mild and severe progression of preexistent cartilage defects. Regatte et $\mathrm{al}^{22}$ report that the range of $\mathrm{T} 2$ values varied from $32 \pm 2$ to $45 \pm 4 \mathrm{~ms}$ and increased with different grades of OA; however, no great differences between $\mathrm{OA}$ groups were demonstrated. We had similar results, but the $\mathrm{T} 2$ values or $\mathrm{T} 2$ value ranges were not identical (Table 1). ${ }^{13,22}$ The explanation might be that the underlying cartilage changes mainly occurred early in the development of the disease and did not progress as much as mechanical degradation of the cartilage. Furthermore, the K-L score criteria had some limitations. ${ }^{41}$ Compared with our previous results, T2 values differed slightly. ${ }^{26} \mathrm{We}$ conclude that $\mathrm{T} 2$ relaxation time was sensitive in the detection of the early stage of $\mathrm{OA}$, which involves the primary degeneration of collagen.

The dGEMRIC technique was also applied in our study, which uses the principle of fixed-charge density and proved to be relatively sensitive to the change of GAGs in cartilage. ${ }^{9,11}$ Within the cartilage, the contrast agent and

Table 3 The correlations among all the influencing factors with Pearson correlation analysis

\begin{tabular}{llllll}
\hline Characteristic & Age & Sex & $\begin{array}{l}\text { TI relaxation } \\
\text { time }\end{array}$ & $\begin{array}{l}\text { T2 relaxation } \\
\text { time }\end{array}$ & $\begin{array}{l}\text { Magnetization } \\
\text { transfer ratio }\end{array}$ \\
\hline Age & $\mathrm{I}$ & $0.629 * *$ & 0.254 & $-0.400 *$ & 0.130 \\
Sex & $0.629 * *$ & $\mathrm{I}$ & -0.143 & $-0.409 *$ & -0.238 \\
TI relaxation time & 0.254 & -0.143 & $\mathrm{I}$ & $-0.31 \mathrm{I}$ & -0.165 \\
T2 relaxation time & $-0.400 *$ & $-0.409 *$ & -0.311 & $\mathrm{I}$ & $0.49 I^{* *}$ \\
Magnetization transfer ratio & 0.130 & -0.238 & -0.165 & $0.49 I^{* *}$ & $\mathrm{I}$ \\
\hline
\end{tabular}

Notes: *Correlation is significant at the 0.05 level (two-tailed). ${ }^{* *}$ Correlation is significant at the 0.01 level (two-tailed). 
Table 4 Accuracy rate of the original grouped cases correctly classified (\%) with discriminative analysis

\begin{tabular}{llllll}
\hline Accuracy rate & $\begin{array}{l}\text { TI relaxation } \\
\text { time }\end{array}$ & Sex & $\begin{array}{l}\text { Magnetization } \\
\text { transfer ratio }\end{array}$ & $\begin{array}{l}\text { T2 relaxation } \\
\text { time }\end{array}$ \\
\hline $81.8 \%$ & $\times$ & $\sqrt{ }$ & $\sqrt{ }$ & $\sqrt{ }$ \\
$81.8 \%$ & $\sqrt{ }$ & $\times$ & $\sqrt{ }$ & $\sqrt{ }$ \\
$87.9 \%$ & $\sqrt{ }$ & $\sqrt{ }$ & $\times$ & $\sqrt{ }$ & $\sqrt{ }$ \\
$87.9 \%$ & $\sqrt{ }$ & $\sqrt{ }$ & $\sqrt{ }$ & $\sqrt{ }$ \\
$90.9 \%$ & $\sqrt{ }$ & $\sqrt{ }$ & $\sqrt{ }$ & $\sqrt{ }$ \\
$93.9 \%$ & $\sqrt{ }$ & $\sqrt{ }$ & $\sqrt{ }$ & $\sqrt{ }$ & $\sqrt{ }$ \\
\hline
\end{tabular}

Notes: $\times$, exclusion of this factor. $\sqrt{ }$, inclusion of this factor.

GAGs interacted and the contrast agent was distributed in an inverse relationship with GAGs. The negatively charged Gd-DTPA ${ }^{2-}$ accumulates with high concentration in areas where there is low GAG content. This leads to reduced $\mathrm{T} 1$ relaxation time; therefore, $\mathrm{T} 1$ relaxation time was used as a quantitative reflection of GAG content in this study. The GAG concentration in the articular cartilage was not consistent, displaying zonal organization with a decrease in GAG content from the deep to the superficial layers of the cartilage. ${ }^{9,16}$ Bashir et $\mathrm{al}^{9}$ report that $\mathrm{T} 1$ relaxation changes distinctly if the GAG content is reduced by $50 \%$.

In our study, the $\mathrm{T} 1$ relaxation times of the three groups (normal, mild OA, and moderate OA) were 481.7 \pm 66.9 , $478.3 \pm 64.5$, and $407.0 \pm 42 \mathrm{~ms}$, respectively, and we found that $\mathrm{T} 1$ relaxation times decreased with the worsening of OA (Table 1). These results indicate that the loss of GAGs increased with the aggravation of cartilage degeneration. Similar results were found in some previous studies. ${ }^{16,26}$ A significant difference was only found between the normal and moderate OA groups. Our data for abnormal cartilage relaxation times were different from those of previous reports. ${ }^{16,42}$ There are several possible reasons, such as that more accurate $\mathrm{T} 1$ relaxation times were obtained with a higher field intensity; that there are differences in contrast administration and imaging protocol, such as the dose of contrast agent and the interval between contrast administration and image acquisition; that there are differences in the mathematical formulae or the parameters of the applied sequence; and that the GAG concentration in cartilage was not uniform, and thus, measurement differences or errors were inevitable.

The GAG content in cartilage was related not only to the cartilage quantity but also to the cartilage quality. ${ }^{43}$ In the mild OA group, the changes of cartilage quantity and cartilage quality may not be great, so the accompanying loss of GAGs was also minimal. A balance between the presence of PG and the rigid collagen network plays a key role in normal cartilage, and insufficient viscoelasticity resulting from low GAG content in the cartilage matrix may cause collagen loss. ${ }^{44}$ In the mild OA group, the balance was neither destroyed nor in the compensated stage, so the $\mathrm{T} 1$ relaxation times were slightly lower than those of the normal cartilage. However, the balance was broken in the late stage of the moderate OA. The reasons mentioned previously may explain why there was no significant difference between the mild OA and normal groups. The relatively small amount of study data is another possible factor. Thus, further investigation is encouraged.

The MT imaging technique is currently a valuable tool for providing the structure and biochemical compositions of cartilage in vivo. ${ }^{18-21}$ The principle of MT's effects is based on the interaction of the free (unbound) water pool and the bound proton pool. The majority of studies report that the collagen component is the most important contributor to the MT effect, ${ }^{18-21,45}$ but Gray et al ${ }^{45}$ also have reported that MT value changes may be a result of physiological or pathophysiological changes in GAG concentration and tissue structure.

Table 5 Hypothetical quantitative and refined grades of osteoarthritis based on the present study

\begin{tabular}{llll}
\hline Characteristics & Normal & Mild & Moderate \\
\hline Age & $23.94-31.27$ & $41.85-53.78$ & $56.79-65.2$ I* $^{\dagger}$ \\
Sex & Male/female & Female $^{\dagger / m a l e}$ & Female $/$ male \\
TI relaxation time & $447.46-516.27$ & $432.12-524.39$ & $362.62-451.3^{*}$ \\
T2 relaxation time & $46.07-48.63$ & $48.01-54.1$ & $49.58-58.22^{*}$ \\
Magnetization transfer ratio & $34.58-36.95$ & $34.72-37.54$ & $33.68-37.19^{*}$ \\
Current K-L grade & 0 & $1-2$ & 3 \\
\hline
\end{tabular}

Notes: *Women were dominant. ${ }^{\dagger 95 \%}$ confidence interval. 
Regatte et $\mathrm{al}^{46}$ report that MTR in bovine patellar cartilage was depth-dependent and was higher in the radial zone. They also explain that the high MTR not only depended on collagen content but may also reflect a number of other parameters. ${ }^{46} \mathrm{In}$ our study, the MTR values were $35.8 \% \pm 4.2 \%, 36.1 \% \pm 3.2 \%$, and $35.4 \% \pm 3.8 \%$, respectively, which are similar to the MTR values in normal cartilage reported by Palmieri et a ${ }^{19}$ and Welsch et $\mathrm{a}^{21}$ but far different than the reported values of $85 \%-86 \%, \pm 1 \%$, by Stanisz et al ${ }^{47}$ and Yao et $\mathrm{al}^{26}$ (Tables 1 and 2). In our present study, no significant differences were found among these three groups regarding MTR (Table 2), which is similar to in Welsch et al ${ }^{21}$ who considered that $\mathrm{T} 2$ decreases with $\mathrm{T} 1$ increases may counterbalance their effects on the MT contrast achieved with Steady-State Free Precession at 7 T. The changes in MTR likely reflect the changes in collagen structure, rather than collagen concentration.$^{48}$ In addition, MTR is susceptible to many factors, such as deviation frequencies, radio-frequency angles, and field intensities. All the influential factors may result in small discrepancies of MTR and may limit its application in cartilage degeneration evaluation. Further studies on the MT technique are needed.

An interesting finding in our study was the positive correlation between T1 relaxation times and MTR values (Tables 2 and 3), although MTR values differ slightly from each other. In our study, to minimize the effect on MT, we chose the proton-density gradient echo sequence for MTC examination, which has been previously recommended. ${ }^{49}$ The results of the present study suggest that the decrease of GAGs on dGEMRIC could also theoretically be reflected on MT imaging, although MTR values changed very slightly. In the cartilage, interchain interactions between the two main compositions of collagen and PGs are possible, and the contributions from PG or other noncollagenous macromolecule content may affect the MT ratios in cartilage structures..$^{50} \mathrm{Ahn}$ et $\mathrm{al}^{51}$ report that three-dimensional MTC had higher specificity in the detection of grade 3 lesions. Koskinen et $\mathrm{al}^{52}$ report that there was a positive correlation between histologically graded cartilage degeneration and T1 relaxation times through a study of 23 cadaveric patellae, and that no correlation was found between cartilage damage and T2 relaxation times. However, the MTR values were influenced by many factors, which contributed to small variations. Therefore, correlation between the $\mathrm{T} 1$ relaxation times and MTR values is possible, and we may postulate that MTR can reflect not only the collagen concentration and structure changes but also the PG content changes in cartilage. Thus, the correlation could possibly be accounted for. The degeneration of cartilage is a complicated and chronic process, and some of the details and exact mechanisms are still unknown and require further exploration.

At this time, the K-L score criteria provide a nonquantitative OA division ${ }^{25}$ based on radiological features such as the formation of osteophytes on the joint margins, periarticular ossicles, narrowing of joint cartilage associated with sclerosis of subchondral bone, and so on. Lack of the quantitative evaluation between normal and OA cartilage is a limitation. Therefore, the pursuit of the combination of quantitative evaluation with K-L scores in the application of early OA is novel and helpful. Thus, in the present study, the application of quantitative parameters with the factors of age and sex to calculate the accuracy of original grouped cases correctly classified with discriminative analysis was investigated. The results were satisfactory, and accuracy rate was high, reaching $93.9 \%$. As seen in Table 4, accuracy was influenced by many factors, including age, sex, T1 relaxation time, T2 relaxation time, and MTR. An interesting finding is that the factors $\mathrm{T} 1$ relaxation and sex played the main role (Table 4). Here, the factor of sex should be emphasized. In the process of OA, sex played an important role, and the female predisposition toward knee OA may, at least in part, be a result of sex differences in cartilage. Even before the onset of clinical knee disease, women display more severe knee degeneration than men. ${ }^{53-55}$ Our results are consistent with previous reports and reconfirm the importance of sex in the development of OA.

The highlight of this study is the combination of the quantitative and nonquantitative evaluation of cartilage. We could possibly conclude that quantitative evaluation with reference to the current K-L score criteria and some relevant patient information may be useful in clinical evaluation in early OA. On the basis of our data, the aim to quantify and refine the current K-L score criteria was expected to be achieved. Thus, we proposed a quantitative and refined grade (Table 5), in which the current K-L score criteria with some quantitative parameters and relative information were newly enrolled. Thus, the superiority of the new hypothetical quantitative and refined grades over the current K-L score criteria is the realization of the quantitative evaluation of the cartilage. The new grade application is expected to provide effective aids in diagnosis, monitoring the changes of the illness and treatment options.

Another significant point is that the present study results can supplement the current three semiquantitative scoring systems used for whole-organ assessment of knee OA: the Whole-Organ Magnetic Resonance Imaging Score, ${ }^{56}$ the Knee Osteoarthritis Scoring System, ${ }^{57}$ and the Boston Leeds 
Osteoarthritis Knee Score. ${ }^{58}$ The main items of the three methods include cartilaginous defects, osteophytes, marrow abnormality, and other such factors, but the methods lack quantitative evaluation and clinical information enrollment, such as that of age and sex.

However, in this study, lack of a severe OA group and lack of age-matched controls were primary limitations. The results may be improved with higher magnetic field intensity. We will continue with further research to perfect our study.

In summary, $\mathrm{T} 1$ relaxation time and $\mathrm{T} 2$ relaxation time are useful biomarkers of early OA. In addition, T2 relaxation time is relatively more sensitive. The usefulness of MTR is relatively limited in the present study, but that situation may change with further studies. Multiple quantitative parameters combined with some relative nonquantitative clinical parameters of patients and K-L score criteria may be useful in the evaluation of early stage of OA in the clinic and may lay the foundation for clinical treatment and follow-up. In addition, the positive linear correlation between $\mathrm{T} 1$ relaxation time and MTR may be a useful finding.

\section{Acknowledgments}

The authors thank Dr Li Chao for her statistical support in the understanding of this paper.

This study was supported by a grant from the National Natural Science Foundation of China (no. 81171312).

\section{Disclosure}

The authors report no conflicts of interest in this work.

\section{References}

1. Lawrence RC, Felson DT, Helmick CG, et al; National Arthritis Data Workgroup. Estimates of the prevalence of arthritis and other rheumatic conditions in the United States. Part II. Arthritis Rheum. 2008;58(1):26-35.

2. Thomas E, Peat G, Croft P. Defining and mapping the person with osteoarthritis for population studies and public health. Rheumatology (Oxford). 2014;53(2):338-345.

3. Oliveria SA, Felson DT, Reed JI, Cirillo PA, Walker AM. Incidence of symptomatic hand, hip, and knee osteoarthritis among patients in a health maintenance organization. Arthritis Rheum. 1995;38(8): 1134-1141.

4. Buckwalter JA, Martin J. Degenerative joint disease. Clin Symp. 1995; 47(2):1-32.

5. Dijkgraaf LC, de Bont LG, Boering G, Liem RS. The structure, biochemistry, and metabolism of osteoarthritic cartilage: a review of the literature. J Oral Maxillofac Surg. 1995;53(10):1182-1192.

6. Buckwalter JA, Mankin HJ. Articular cartilage: degeneration and osteoarthritis, repair, regeneration, and transplantation. Instr Course Lect. 1998;47:487-504.

7. van de Loo AA, Arntz OJ, Otterness IG, van den Berg WB. Proteoglycan loss and subsequent replenishment in articular cartilage after a mild arthritic insult by IL-1 in mice: impaired proteoglycan turnover in the recovery phase. Agents Actions. 1994;41(3-4): 200-208.
8. Taylor C, Carballido-Gamio J, Majumdar S, Li X. Comparison of quantitative imaging of cartilage for osteoarthritis: T2, T1rho, dGEMRIC and contrast-enhanced computed tomography. Magn Reson Imaging. 2009;27(6):779-784.

9. Bashir A, Gray ML, Boutin RD, Burstein D. Glycosaminoglycan in articular cartilage: in vivo assessment with delayed Gd(DTPA)(2-)enhanced MR imaging. Radiology. 1997;205(2):551-558.

10. Koster IM, Oei EH, Hensen JH, et al. Predictive factors for new onset or progression of knee osteoarthritis one year after trauma: MRI follow-up in general practice. Eur Radiol. 2011;21(7):1509-1516.

11. Fleming BC, Oksendahl HL, Mehan WA, et al. Delayed GadoliniumEnhanced MR Imaging of Cartilage (dGEMRIC) following ACL injury. Osteoarthritis Cartilage. 2010;18(5):662-667.

12. Regatte RR, Akella SV, Wheaton AJ, et al. 3D-T1rho-relaxation mapping of articular cartilage: in vivo assessment of early degenerative changes in symptomatic osteoarthritic subjects. Acad Radiol. 2004;11(7):741-749.

13. Li X, Benjamin Ma C, Link TM, et al. In vivo $\mathrm{T}(1$ rho) and $\mathrm{T}(2)$ mapping of articular cartilage in osteoarthritis of the knee using 3 T MRI. Osteoarthritis Cartilage. 2007;15(7):789-797.

14. Staroswiecki E, Bangerter NK, Gurney PT, Grafendorfer T, Gold GE, Hargreaves BA. In vivo sodium imaging of human patellar cartilage with a 3D cones sequence at $3 \mathrm{~T}$ and $7 \mathrm{~T}$. J Magn Reson Imaging. 2010;32(2):446-451.

15. Jessel RH, Zilkens C, Tiderius C, Dudda M, Mamisch TC, Kim YJ. Assessment of osteoarthritis in hips with femoroacetabular impingement using delayed gadolinium enhanced MRI of cartilage. J Magn Reson Imaging. 2009;30(5):1110-1115.

16. Bashir A, Gray ML, Hartke J, Burstein D. Nondestructive imaging of human cartilage glycosaminoglycan concentration by MRI. Magn Reson Med. 1999;41(5):857-865.

17. Apprich S, Mamisch TC, Welsch GH, et al. Quantitative T2 mapping of the patella at $3.0 \mathrm{~T}$ is sensitive to early cartilage degeneration, but also to loading of the knee. Eur J Radiol. 2012;81(4): e438-e443.

18. Wolff SD, Chesnick S, Frank JA, Lim KO, Balaban RS. Magnetization transfer contrast: MR imaging of the knee. Radiology. 1991;179(3):623-628.

19. Palmieri F, De Keyzer F, Maes F, Van Breuseghem I. Magnetization transfer analysis of cartilage repair tissue: a preliminary study. Skeletal Radiol. 2006;35(12):903-908.

20. Welsch GH, Apprich S, Zbyn S, et al. Biochemical (T2, T2* and magnetisation transfer ratio) MRI of knee cartilage: feasibility at ultrahigh field (7 T) compared with high field (3 T) strength. Eur Radiol. 2011;21(6):1136-1143.

21. Welsch GH, Trattnig S, Scheffler K, et al. Magnetization transfer contrast and T2 mapping in the evaluation of cartilage repair tissue with 3 T MRI. J Magn Reson Imaging. 2008;28(4):979-986.

22. Regatte RR, Akella SV, Lonner JH, Kneeland JB, Reddy R. T1rho relaxation mapping in human osteoarthritis (OA) cartilage: comparison of T1rho with T2. J Magn Reson Imaging. 2006;23(4):547-553.

23. Gold GE, Hargreaves BA, Stevens KJ, Beaulieu CF. Advanced magnetic resonance imaging of articular cartilage. Orthop Clin North Am. 2006;37(3):331-347, vi. vi.

24. Bellamy N, Buchanan WW, Goldsmith CH, Campbell J, Stitt LW. Validation study of WOMAC: a health status instrument for measuring clinically important patient relevant outcomes to antirheumatic drug therapy in patients with osteoarthritis of the hip or knee. J Rheumatol. 1988;15(12):1833-1840.

25. Kellgren JH, Lawrence JS. Radiological assessment of osteo-arthrosis. Ann Rheum Dis. 1957;16(4):494-502.

26. Yao $\mathrm{W}, \mathrm{Qu} \mathrm{N}, \mathrm{Lu} \mathrm{Z}$, Yang S. The application of $\mathrm{T} 1$ and $\mathrm{T} 2$ relaxation time and magnetization transfer ratios to the early diagnosis of patellar cartilage osteoarthritis. Skeletal Radiol. 2009;38(11):1055-1062.

27. Radin EL, Rose RM. Role of subchondral bone in the initiation and progression of cartilage damage. Clin Orthop Relat Res. 1986;(213):34-40. 
28. Plewes DB, Kucharczyk W. Physics of MRI: a primer. J Magn Reson Imaging. 2012;35(5):1038-1054.

29. Katz JD, Agrawal S, Velasquez M. Getting to the heart of the matter: osteoarthritis takes its place as part of the metabolic syndrome. Curr Opin Rheumatol. 2010;22(5):512-519.

30. Mosher TJ, Dardzinski BJ. Cartilage MRI T2 relaxation time mapping: overview and applications. Semin Musculoskelet Radiol. 2004;8(4):355-368.

31. David-Vaudey E, Ghosh S, Ries M, Majumdar S. T2 relaxation time measurements in osteoarthritis. Magn Reson Imaging. 2004; 22(5):673-682.

32. Keenan KE, Besier TF, Pauly JM, et al. Prediction of glycosaminoglycan content in human cartilage by age, T1 $\rho$ and T2 MRI. Osteoarthritis Cartilage. 2011;19(2):171-179.

33. Nishioka H, Hirose J, Nakamura E, et al. T1 $\rho$ and T2 mapping reveal the in vivo extracellular matrix of articular cartilage. J Magn Reson Imaging. 2012;35(1):147-155.

34. Wong CS, Yan CH, Gong NJ, Li T, Chan Q, Chu YC. Imaging biomarker with T1 $\rho$ and T2 mappings in osteoarthritis - in vivo human articular cartilage study. Eur J Radiol. 2013;82(4):647-650.

35. Mosher TJ, Dardzinski BJ, Smith MB. Human articular cartilage: influence of aging and early symptomatic degeneration on the spatial variation of T2 - preliminary findings at $3 \mathrm{~T}$. Radiology 2000;214(1):259-266.

36. Cha JG, Lee JC, Kim HJ, et al. Comparison of MRI T2 relaxation changes of knee articular cartilage before and after running between young and old amateur athletes. Korean J Radiol. 2012;13(5):594-601.

37. Kim T, Min BH, Yoon SH, et al. An in vitro comparative study of $\mathrm{T} 2$ and $\mathrm{T} 2 *$ mappings of human articular cartilage at 3-Tesla MRI using histology as the standard of reference. Skeletal Radiol. 2014;43(7):947-954.

38. Wei ZM, Du XK, Huo TL, et al. Quantitative T2 mapping evaluation for articular cartilage lesions in a rabbit model of anterior cruciate ligament transection osteoarthritis. Chin Med J (Engl). 2012;125(5): 843-850.

39. Dunn TC, Lu Y, Jin H, Ries MD, Majumdar S. T2 relaxation time of cartilage at MR imaging: comparison with severity of knee osteoarthritis. Radiology. 2004;232(2):592-598.

40. Prasad AP, Nardo L, Schooler J, Joseph GB, Link TM. T1 $\rho$ and T2 relaxation times predict progression of knee osteoarthritis. Osteoarthritis Cartilage. 2013;21(1):69-76.

41. Sharma L. Epidemiology of osteoarthritis. In: Moskowitz RW, Howell DS, Altman RD, Buckwalter JA, Goldberg VM, eds. Osteoarthritis: diagnosis and medical/surgical management. 3rd ed. Philadelphia, Pa: Saunders; 2001:3-28.

42. Burstein D, Velyvis J, Scott KT, et al. Protocol issues for delayed Gd(DTPA)(2-)-enhanced MRI (dGEMRIC) for clinical evaluation of articular cartilage. Magn Reson Med. 2001;45(1):36-41.

43. Tiderius CJ, Olsson LE, Nyquist F, Dahlberg L. Cartilage glycosaminoglycan loss in the acute phase after an anterior cruciate ligament injury: delayed gadolinium-enhanced magnetic resonance imaging of cartilage and synovial fluid analysis. Arthritis Rheum. 2005;52(1):120-127.
44. Poole AR, Nelson F, Dahlberg L, et al. Proteolysis of the collagen fibril in osteoarthritis. Biochem Soc Symp. 2003;(70):115-123.

45. Gray ML, Burstein D, Lesperance LM, Gehrke L. Magnetization transfer in cartilage and its constituent macromolecules. Magn Reson Med. 1995;34(3):319-325.

46. Regatte RR, Akella SV, Reddy R. Depth-dependent proton magnetization transfer in articular cartilage. $J$ Magn Reson Imaging. 2005;22(2):318-323.

47. Stanisz GJ, Odrobina EE, Pun J, et al. T1, T2 relaxation and magnetization transfer in tissue at 3T. Magn Reson Med. 2005;54(3):507-512.

48. Venn M, Maroudas A. Chemical composition and swelling of normal and osteoarthrotic femoral head cartilage. I. Chemical composition. Ann Rheum Dis. 1977;36(2):121-129.

49. Vahlensieck M, Gieseke J, Träber F, Schild H. [Magnetization transfer contrast (MTC) - which is the most MTC-sensitive MRI sequence?] Rofo. 1998;169(2):195-197.

50. Lattanzio PJ, Marshall KW, Damyanovich AZ, Peemoeller H. Macromolecule and water magnetization exchange modeling in articular cartilage. Magn Reson Med. 2000;44(6):840-851.

51. Ahn JM, Kwak SM, Kang HS, et al. Evaluation of patellar cartilage in cadavers with a low-field-strength extremity-only magnet: comparison of MR imaging sequences, with macroscopic findings as the standard. Radiology. 1998;208(1):57-62.

52. Koskinen SK, Komu M, Aho HJ, Kormano M. MR imaging of patellar cartilage degeneration at 0.02 T. Study of 23 cadaveric patellae. Acta Radiol. 1991;32(6):514-517.

53. Hame SL, Alexander RA. Knee osteoarthritis in women. Curr Rev Musculoskelet Med. 2013;6(2):182-187.

54. Muehleman C, Margulis A, Bae WC, Masuda K. Relationship between knee and ankle degeneration in a population of organ donors. $B M C$ Med. 2010;8;8:48. http://dx.doi.org/10.1186/1741-7015-8-48

55. Hanna FS, Teichtahl AJ, Wluka AE, et al. Women have increased rates of cartilage loss and progression of cartilage defects at the knee than men: a gender study of adults without clinical knee osteoarthritis. Menopause. 2009;16(4):666-670.

56. Peterfy CG, Guermazi A, Zaim S, et al. Whole-Organ Magnetic Resonance Imaging Score (WORMS) of the knee in osteoarthritis. Osteoarthritis Cartilage. 2004;12(3):177-190.

57. Kornaat PR, Ceulemans RY, Kroon HM, et al. MRI assessment of knee osteoarthritis: Knee Osteoarthritis Scoring System (KOSS) - interobserver and intra-observer reproducibility of a compartment-based scoring system. Skeletal Radiol. 2005;34(2):95-102.

58. Hunter DJ, Lo GH, Gale D, Grainger AJ, Guermazi A, Conaghan PG. The reliability of a new scoring system for knee osteoarthritis MRI and the validity of bone marrow lesion assessment: BLOKS (Boston Leeds Osteoarthritis Knee Score). Ann Rheum Dis. 2008;67(2):206-211.
Clinical Interventions in Aging

\section{Publish your work in this journal}

Clinical Interventions in Aging is an international, peer-reviewed journal focusing on evidence-based reports on the value or lack thereof of treatments intended to prevent or delay the onset of maladaptive correlates of aging in human beings. This journal is indexed on PubMed Central, MedLine,
CAS, Scopus and the Elsevier Bibliographic databases. The manuscript management system is completely online and includes a very quick and fair peer-review system, which is all easy to use. Visit http://www.dovepress. com/testimonials.php to read real quotes from published authors. 